\title{
Heuristic Algorithm for Forming the Service Schedule for the Multistage Systems with a Generalized Resource Criterion
}

\author{
S. A. Oleinikova ${ }^{1 *}$, O. Ya. Kravets ${ }^{1}$, V. D. Sekerin ${ }^{2}$, A. E. Gorokhova ${ }^{2}$, V. I. Gayduk ${ }^{3}$ \\ ${ }^{I}$ Voronezh Technical State University, Voronezh, Russian Federation, prosp. Moskovskij 14, Voronezh, 394026, Russia \\ ${ }^{2}$ Moscow Polytechnic University, B. Semenovskaya Street 38, Moscow, 107023, Russia \\ ${ }^{3}$ Kuban State Agrarian University, 13 Kalinina Str., Krasnodar, 350044, Russia \\ *Corresponding author E-mail: oleinikova.s.a@mail.ru
}

\begin{abstract}
The paper suggests one of the approaches to solving the problem of planning multi-stage service systems, the distinguishing feature of which is the availability of time constraints and a resource criterion. At the heart of the approach lie heuristics, which make it possible to obtain a suboptimal solution in an acceptable time. Based on these heuristics, the process of making decisions about assigning jobs to a given time interval, determining the optimal time interval for the return, and estimating the remaining service time are carried out.
\end{abstract}

Keywords: heuristics, algorithm, multi-stage service systems, generalized resource criterion, time constraints.

\section{Introduction}

The efficiency of the functioning of the servicing systems is largely determined by the quality of scheduling. The necessity of processing of multiple applications with individual service options, including the solution of a large number of interdependent tasks, time and resource constraints, the presence of several service centers cause the development of algorithms and software systems that allow you to obtain a schedule of works which is optimal from the point of view of a given criterion, and to automate the process of its formation.

This problem belongs to the class of NP-complete problems of scheduling theory. At present time, many problems in this field have already been investigated and widely studied. The algorithms for solving these problems are based on the CMP (Critical Path Method) approach, which is a technique for determining the start time of works with the presence of the constraints on mutual dependence and without taking into account resource constraints. This approach is described in detail in [1-5]. However, in practice, resource constrained project scheduling problem is more in demand. There exist many algorithms for solving this problem [6-9]. The most well-known are those algorithms that are oriented toward the speedy completion of all works. But for many systems, time does not appear as a criterion, but as a limitation, which complicates the planning process. Therefore, there is a need to obtain the methods that take into account these features.

This paper is devoted to the development of an algorithm for solving the project management problem with resource and time constraints. The first part provides a mathematical description that takes into account the time and resource criteria and aims to optimize the amount of used resources from the point of view of the decision-maker. A generalized algorithm with a return based on the developed mathematical apparatus is presented in the second part. The third part is devoted to detailing such stages of the algorithm as the choice of a possible stage for a return, the lower estimate of the completion time of the project, and the determination of the next planning moment.

\section{The Mathematical Statement of the Prob- lem}

The problem of forming of the scheduling chart for multi-serving system is considered. The incoming flow is applications that require for their service to perform a plurality of mutuallydependent works. The system consists of several centers:

$$
S=\left(S_{1}, \ldots, S_{L}\right)
$$

Each center $j(j=1, \ldots, L)$ is specialized in a certain kind of service and has a plurality of several types of resources:

$$
R_{j}=\left(R_{j 1}, \ldots, \mathrm{R}_{\mathrm{jk}_{\mathrm{j}}}\right)
$$

Here $\mathrm{kj}$ - the number of types of resources at the center $\mathrm{j}, \mathrm{Rj} 1, \ldots$, $\mathrm{Rjkj}$ - the amount of each type of resource.

Each application Pm coming to the input of the model is a set of mutually-dependent works. In addition to work, the application is defined by time Tm, for which it is necessary to complete the service:

$$
P_{m}=P_{m}\left(T_{m}, \mathrm{~W}_{\mathrm{m}}\right)
$$

Every work $w_{i} \in W_{m}$ is defined by the following set of characteristics:

$w_{i}=w_{i}\left(S_{i}, d u r_{i}, R_{i}, \operatorname{Pr}_{i}\right)$

Here $\mathrm{Si}$ - the center in which the work $\mathrm{i}$ is performed; duri - the approximate time of service; $\mathrm{Ri}=(\mathrm{Ri} 1, \ldots, \mathrm{Riki})$ - the necessary resources; Pri - multiplicity of works what immediately preceding the implementation of the work $i$. 
It is necessary to determine the start time of each operation wi.beg such a way as to optimize the functioning of the system taking into account the restrictions on the available resources and the mutual dependence of works. As an optimization criterion a generalized resource criterion is considered. Let the function $\mathrm{fj}(\mathrm{t})$ determines the most appropriate level of resources that is used at the time $t$ in the center $\mathrm{j}$.

$$
f_{j}(t)=\left(f_{j 1}(t), \ldots, f_{j k j}(t)\right)
$$

Loading of the system by servicing the requests received earlier in the center $\mathrm{j}$ is described by the vector:

$$
R_{\text {usd } j}(t)=\left(R_{\text {usd } j 1}(t), \ldots, R_{u s d j k j}(t)\right)
$$

Then, the objective function for each center will minimize the deviation of the actual volume of resources, resulting in solving the planning problem, from a given in that moment volume:

$$
\left\{\begin{array}{l}
\sum_{t} \sum_{i=1}^{k_{1}}\left(\sum_{j: w_{j} \in W_{t}} w_{j} \cdot r_{i}+R_{\mathrm{usd} 1 \mathrm{ki}}(t)-f_{1 i}(t)\right)^{2} \rightarrow \min \\
\cdots \\
\sum_{t} \sum_{i=1}^{k_{L}}\left(\sum_{j: w_{j} \in W_{t}} w_{j} \cdot r_{i}+R_{\mathrm{usd} \mathrm{L} \mathrm{ki}}(t)-f_{L i}(t)\right)^{2} \rightarrow \min
\end{array}\right.
$$

Here $\mathrm{t}$ is the current planning moment; $\mathrm{kj}$ - the number of resources used to perform the works in the center $\mathrm{j}$; $\mathrm{i}$ - $\mathrm{i}$-th kind of resources; wj.ri - the amount of resources of type $i$ that are required to perform the work wj; fi and Rusd i are determined formulas (5) and (6), respectively. Thus, the criterion (7) reflects the need at each time create a schedule so that the volume of each type of resource involved was close to the reference value.

This objective function is selected for the following reasons. Firstly, there exist the tasks for which it is necessary to complete the project at a given time. At the same time, the implementation of the project in the shortest possible time (within specified constraints) does not allow to obtain the best solution. Moreover, it is not always appropriate for many such applications. Secondly, such loading of system allows to increase its reserve capacity in general It is also necessary to suggest the emergence of new unplanned projects that require a speedy service in a short time. When creating a schedule for the projects received before in terms of early completion, the system may not have enough resources that will ensure the timely implementation of the new project. In this connection, it may be necessary to reschedule the works, which is already scheduled start time, at a later date, i.e., to amend the existing schedule. In the presence of a mutual dependence between the works, as well as time and resource constraints, this problem may require significant time and algorithmic costs. In addition, the variation of the functions $\mathrm{fj}$ can provide a solution to the original problem in a variety of conditions. If this function coincides with the function (6), it will provide an opportunity to the maximum load of the system. If the function does not change over time, it will get a criterion for uniform loading.

Thus, the use of the generalized criterion of resource will not only take into account the specifics of a number of systems that do not require the completion of service of their applications as soon as possible, but also efficient use of the resource potential for such systems.

Restrictions

The start time of works must be determined in conditions of resource constraints. Resource limitations imply that for each center $\mathrm{j}$ the set of jobs Wt (which will be assigned the start time t) can be formed only in such a way that the total amount of resources did not exceed the total amount of resources of this type Rji. Moreover, it is necessary to take into account the resources used to carry out the work planned earlier. Thus, the restrictions on resources will be written in the form:

$$
\sum_{w_{m} \in W_{t}} w_{m} \cdot r_{i}+R_{\text {usd ji }}(t) \leq R_{\mathrm{ji}}(t), \mathrm{i}=1, \ldots, \mathrm{k}_{\mathrm{j}} .
$$

In addition, it is necessary take into account the restriction on mutual dependence of works:

$w_{i} \cdot t_{\text {beg }} \geq w_{k} \cdot t_{\text {beg }}+w_{k} \cdot d u r, \forall w_{k} \in \operatorname{Pr}_{i}$

Here wj.tbeg - the start time of the work wi; wj.dur - the duration of its work.

Another important feature of the task is the restriction on the duration of the project. This restriction can be described with the help of the following inequality:

$T_{i_{-} \text {fact }} \leq T_{i}$

Here Ti_fact is the actual time spent servicing the application i. It can be determined by the formula:

$$
T_{i_{-} f a c t}=\max _{j}\left(w_{j} \cdot t_{b e g}+w_{j} \cdot d u r\right) .
$$

Thus, for each project work, it is necessary to find the starting time wi.tbeg, in which the criteria (7) tend to a minimum and the time and resource constraints (10) and (8), as well as restrictions on the mutual dependence of works (9), take place.

\section{A Generalized Algorithm of Solving the Problem}

Investigated problem belongs to the class of project management tasks. By now this area is sufficiently explored and many partial solutions have already been received. The classical problem is to create a schedule with the requirement of a speedy completion of the project and the resources constraints. The algorithm of its solution is based on the serial assignment a time for works with the smallest time margin. The following features distinguish the studied problem from existing analogues:

- time constraints allowing definitely conclude on the admissibility of the found solution, as a rule, only after all the planning stages;

- the generalized resource criterion (7), which does not allow to plan the maximum of works at a given moment of time and, thereby, reduce the probability of violation of time constraints;

- the presence of the several centers, each of which has its own resources and is characterized by a certain workload at the time $\mathrm{t}$;

- the presence of several types of resources at each center;

- the presence of a schedule at the time of receipt of the application.

The first three features have a direct impact on the general algorithm of the problem; the others determine the specifics of the solution at each time interval.

Consider the specifics of these restrictions in details. The presence of time constraints in conjunction with the resource criterion does not allow to assert that the entire planning will take place without refunds. In this case it may be necessary to change the solutions chosen in the previous stages. This is due to the fact that it is usually possible to determine the fulfillment or non-fulfillment of time constraints only at the end of the planning process. In particu- 
lar, if, when making a decision, we get a negative temporary reserve for some work, it means violation of time limits. This situation requires the cancellation of the found solution and return to one of the previous planning stages.

Given the availability of several service centers, and also taking into account the need to get a solution that is close to optimal, within an acceptable time, it is advisable to parallelize the main stages of the algorithm that are performed in each center to determine the start time of works, taking into account resource constraints. In particular, the process of building a work schedule at this point in time must be parallelized. Since these works can be considered independent of each other, the parallel construction of the schedule will reduce the time spent on planning. Further, it is necessary to provide all planning centers with data on jobs whose start has been postponed to a later time. This will allow you to adjust the early start of dependent works.

Thus, the generalized algorithm for solving the problem will consist of the following stages:

- a preliminary stage, allowing to calculate earlier and later time of the beginning of all works, as well as their temporary reserves;

- the main part of the planning, which determines the start time of works on a given time interval;

- estimate the time of completion of the project;

- possible transition to one of the previous steps to adjust the schedule (in the case of non-fulfillment of time constraints).

The algorithm will look like this (Figure 1).

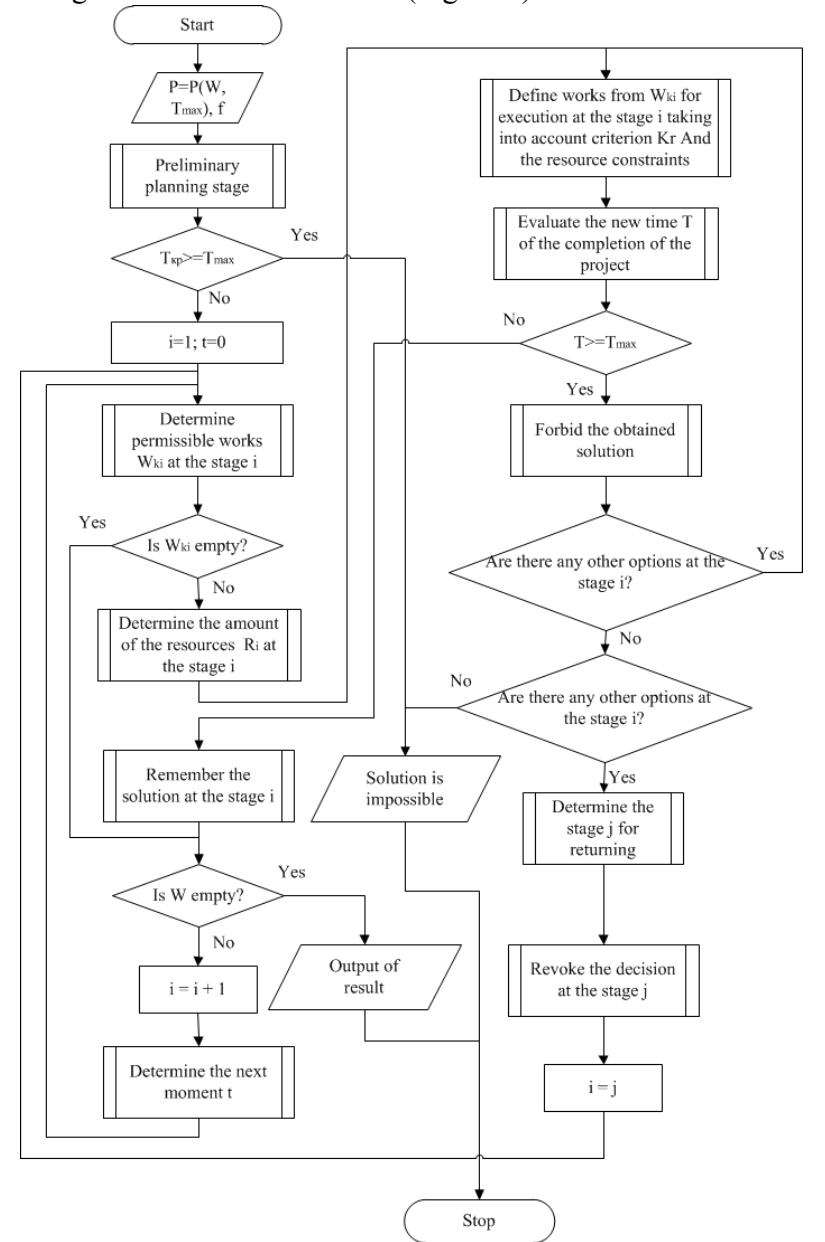

Fig. 1: Generalized algorithm for creating a service schedule

The general idea of the algorithm is as follows. At each stage $i$, the formation of a graph according to criterion (7) and resource constraints (8) is carried out. Since time constraints can be checked only at the end of the planning process, at this stage it is possible to obtain only a lower estimate of this quantity. If it exceeds a critical time, it means violation of time constrains and, as a conse- quence necessity to return to one of the previous stages. Starting from this stage it is required to make a schedule of works anew.

\section{Algorithmic Detailing}

Let's consider the main stages of the algorithm in details. The initial data is a project with a set of works defining it as well as the amount of resources that is most preferable from the perspective of the user. It is also assumed that there is a schedule for servicing applications that have been submitted earlier.

The stage of the preliminary schedule determines the early and late start time of all works without taking into account the amount of resources. The algorithms for determining these characteristics are set forth in [1]. If, in doing so, the time found is greater than the critical value Tcr, then it is not possible to generate a schedule for the given time limits. Otherwise, a further process of scheduling takes place.

Under the set of admissible works Wki in stage i, it means all the works whose early start time allows them to be performed in stage i. If this set is not empty, then the amount of resources necessary to perform all these works is determined. The main step of the algorithm is to select the works from the set Wki to execute at a given time.

The criterion of optimization will be of fundamental importance for the selection of works. In particular, if the criterion for early completion is considered, then at any time point it is necessary to determine the time of performance of works based on their temporary reserve (the work with a minimum reserve should be performed first). In the presence of the cost criterion it is advisable to plan the works so as to get the most profit or a minimum fine. The presence of a generalized resource criterion considered in this task requires the formation of a schedule in such a way that the total amount of resources used reaches a certain standard from the point of view of the user's result.

Since the task is solved in the presence of time constraints, after the next planning, the implementation of these restrictions is carried out. Since the exact value of the service time of the application will be known only after the completion of the schedule formation, at each planning stage will be used a lower estimate of this quantity. In case of violation of restrictions, it is necessary to prohibit this decision, and to determine another planning variant for this interval. If none of the options allows you to do the works in a given time, then there is a transition to one of the previous steps to correct the previously generated schedule. Starting from this stage it is necessary to form other variants of the schedule.

The greatest interest in the algorithm shown in fig. 1, represent the following steps:

- determining the set of works Wki for execution at the stage i;

- evaluation of the project completion time;

- selection the stage for the return (in the case of violation of time constraints).

In case of non-fulfillment of time constraints, it is necessary to return to any of the previous steps to change the schedule on it and to create a new schedule, starting from this time. Let us find the optimal value of the number of stage 1 to return [10].

Suppose that at some stage $t$ the works $1,2, \ldots, j-1$ are planned. The ability to perform the job $\mathrm{j}$ is checked by means of inequalities:

$$
\left\{\begin{array}{l}
R_{1}-R_{1 \_u s d}>w_{j} \cdot r_{1} \\
R_{2}-R_{2 \_u s d}>w_{j} \cdot r_{2} \\
\cdots \\
R_{m}-R_{m_{-} u s d}>w_{j} \cdot r_{m}
\end{array}\right.
$$

If at least one inequality is not met, then it means that the resources do not allow the execution of the work $\mathrm{j}$. 
Suppose that all inequalities (12) are satisfied. Let us introduce the vector

$$
\operatorname{add}(t)=\left(\operatorname{add}_{1}(t), \ldots, a d d_{m}(t)\right)
$$

each element of which shows an additional reserve for performing another work $\mathrm{j}$ on the given time interval, taking into account the resources $\mathrm{k}, \mathrm{k}=1, \ldots, \mathrm{m}$ :

$$
a d d_{k}(t)=R_{k}-R_{k_{-} u s d}(t)-w_{j} . r_{k}, k=1, \ldots, m .
$$

This vector will be negative in those cases when a return to this interval is impractical (all jobs that could be planned - planned or when adding another work, one of the limitations (8) is violated). As a stage for the return, it is advisable to select the stage with the largest reserve. Since the reserve is determined by a multidimensional vector, it is necessary to determine how this choice will be determined.

Let us introduce the concept of the determining type of resources. The defining type of resource is the type of resource that requires the greatest amount of time to complete all work (taking into account the available schedule). To estimate this time use the following approach. Let it be necessary to perform all the works wi from the set Wleft. The total volume of these works for the j-th type of the resources will be determined as follows:

$$
V_{\text {left } j}=\sum_{i \in W_{\text {left }}} w_{i} \cdot d u r \cdot w_{i} \cdot r_{j} .
$$

Here $\mathrm{j}$ - the number of the resource type; wi.dur - the duration of the work wi; wi.rj - the amount of resources of type $j$ that are required to perform the work wi;

Let's find the lower estimate of the duration, which is necessary for performing a multitude of jobs that require a resource of type $j$ Vleft $j$. It will be equal to the ratio of the total volume Vleft $j$ to the volume $\mathrm{Rj}$ of available resources of type $\mathrm{j}$ :

$$
T_{\text {left } j}=\frac{V_{\text {left_j}}}{R_{j}}
$$

Such time will be spent in the event that all the works are assigned such a maintenance schedule in order to use the entire allowable amount of resources at any time.

In this case, first it's need to find the determining type of resources, then select an interval where the reserve for this type of resource is maximal. Let us formulate the corresponding heuristic. Heuristic. Let at some step the violation of time constraints (10) was detected. Then, as a stage to return it is advisable to choose a time for which the vector add will be a solution of the two-stage problem:

1. Find the determining type of resources $\mathrm{j}$, which is the most time-consuming:

$$
T_{\text {left_j }} \rightarrow \max _{1 \leq j \leq m}
$$

2. Determine the time $\mathrm{t}$ for which the reserve for this type of resource is the largest:

$$
\left\{\begin{array}{l}
\operatorname{add}_{j}(t) \rightarrow \max \\
\operatorname{add}_{j}(t) \geq 0
\end{array}\right.
$$

Here the time Tleft $\mathrm{j}$ is determined using the formula (15); vector add is determined using the formula (13).
This choice allows minimally degrade the value of the objective function when adding a new work at the selected step. In the event that there are several such intervals, it is advisable to select an earlier interval.

One of the stages of the algorithm is the determination of the next planning moment. The problem can be formulated as follows. Suppose that at a certain time interval i work planning process is completed. It is necessary to determine the next closest time to form a work schedule.

To solve the problem it is required to determine the set of unfinished works Wn_com. Obviously, the next planning moment will arise in the case of releasing the resources needed to perform the works. Since the resources will be freed if any work from the set Wn_com is completed, it is necessary to find just such time. Therefore, such a moment can be defined as the smallest of the durations of unfinished works:

$t_{\text {next }}=t+\min _{i \in W n \_c o m}\left(w_{i} \cdot d u r\right)$

Here $t$ is the current time; tnext - the next time; Wn_com - is the set of works, which are already started, but not yet completed by the time $\mathrm{t}$.

To complete the description of the generalized algorithm presented in Figure 1, it is necessary to consider the approach to estimating the completion time of the project. For its formation, we again use the defining type of resources, since it is assumed that it will influence the assessment to the greatest extent. Then the time can be estimated as follows:

$$
T_{\text {left }}=T_{\text {cur }}+\max _{1 \leq j \leq m}\left(T_{\min _{-} j}\right)
$$

Here Tleft - the lower estimate of the remaining service time ; Tcur - the current time; Tmin $j$ - the lower estimate of the time remaining until the end of service, calculated by formula (15).

In the event that at every moment of time, starting from Tcur, all available resources will be used, we can get the exact value of the remaining time. Otherwise, this will be a lower estimate, indicating the minimum required time for the fulfillment of all the works.

\section{Conclusion}

The aim of the work was the development of an approach that allows to create a schedule for fulfilling orders in multiphase servicing systems with time constraints and resource criteria for an acceptable time. To achieve this goal, the following tasks have been accomplished:

1. A mathematical model of the system that takes into account the constraints on mutual dependence of works, as well as time and resource constraints, and allows to optimize the process of its functioning from the point of view of the generalized resource criterion is presented.

2. A generalized algorithm for the formation of a service schedule, taking into account the existence of mutually dependent works and time constraints is developed. A feature of the algorithm is the possibility of its application for solving a whole class of planning problems with the above features.

3. The details of the algorithm, describing in general the decision rules for determining the set of works to be performed at a given time, determining the stage for the return, the lower estimate of the length of the service, and determining the next planning moment are proposed.

The next task is the development of a detailed heuristic algorithm that allows solving the problem of partitioning a set of works Wki, the early start time of which allows them to be executed at time i, into two subsets: Wi, which will be executed on the i-th time interval, and $\mathrm{Wi}+1$, which will need to be transferred to the following periods. 


\section{References}

[1] Ahuja HN, Dozzi SP \& Abourizk SM (1994), Project Management: Technics in planning and controlling construction project. Second Edition, New-York: John Willey\&Sons, 509.

[2] Moder JJ, Phillips CR \& Davis EW (1983), Project managemen with CPM, PERT and precedence diagramming, New-York: VanNostrand Reinhold, 389.

[3] Doneva R, Gaftandzhieva S \& Totkov G (2016), FETCH Project: The Maturity of Quality Management Through Dynamic Evaluation of the Project Progress. International Journal on Information Technologies and Security, 3(8), 29-38.

[4] Golenko-Ginzburg D \& Gonik A (1997), Project planning and controlling by stochastic network models. Managing and Modeling Complex Projects, 17, 21-43.

[5] Weglarz J (1999), Project Scheduling - Recent Models, Algorithms and Applications. Boston: Kluwer Academic Publishers, 535.

[6] Demeulemeester EL \& Herroelen WS (2002), Project Scheduling. A research Handbook, New York, Boston, Dordrecht, London, Moscow: Kluwer Academic Publishers, 675

[7] Neumann K, Schwindt C \& Zimmermann J (2003), Project scheduling with time windows and scarce resources. 2 dn Edition, Berlin: Springer, 389.

[8] Blanco L \& Caramia M (2008), A new approach for the Project Scheduling problem with generalized precedence relations. Proceedings of the $11^{\text {th }}$ International Workshop on Project Management and Scheduling, Istanbul, 35-39.

[9] Artigues C, Demassey S \& Neron E (2010), Resource-Constrained Project Scheduling: Models, Algorithms, Extensions and Applications. Willey Online Library, 308.

[10] Oleynikova SA (2014), Algorithm for determing the point of return for scheduling tasks with time constraints and criterion of uniform load. Modern Informatization Problems: Proceedings of the XIX-th International Open Science Conference, Yelm, USA, 67-70. 